\title{
109. Small Earth Strains as Detected by Electric Resistivity Measurements
}

\author{
By Tsuneji Rikitake*) and Yoshio Yamazaki*) \\ (Comm. by Chuji TsuboI, M.J.A., June 12, 1967)
}

Introduction. The 5-year plan for earthquake prediction research (Rikitake, 1966), now under way in Japan, puts much stress on detecting small earth strain which possibly preceeds the occurrence of large earthquakes. In addition to the existing observation techniques using tiltmeters and extensometers, a method of detecting earth strain by measuring changes in earth resistivity has recently been developed by the writers. This report aims at briefly describing the measuring technique as well as the result obtained in the first field experiment.

Laboratory work. Yamazaki $(1965,1966)$ conducted a series of laboratory experiments on changes in electric resistivity of sedimentary rocks caused by mechanical strain. One of the most remarkable facts found by the experiments is that, for some rock specimens, the absolute value of the rate of resistivity change $(\Delta \rho / \rho)$ is far greater than the uniaxial compressional strain $(\Delta L / L)$ given to the specimens. It is also found that resistivity of these rocks always decreases when they are compressed, so that $\Delta \rho / \Delta L$ is always positive.

Fig. 1 shows the relation between $(\Delta \rho / \rho) /(\Delta L / L)$ and $\Delta L / L$ for a lapilli tuff specimen from Aburatsubo, Kanagawa Prefecture which contains $18 \%$ water in weight. It is observed in the figure that the rate of resistivity change is larger than linear strain by a factor as much as of 300 or even more when the strain is about $10^{-5}$. This is one of the typical examples showing this characteristic behaviour of rocks.

It is not quite clear why the rate of resistivity change for lapilli tuff specimen is extremely large for a range of strain

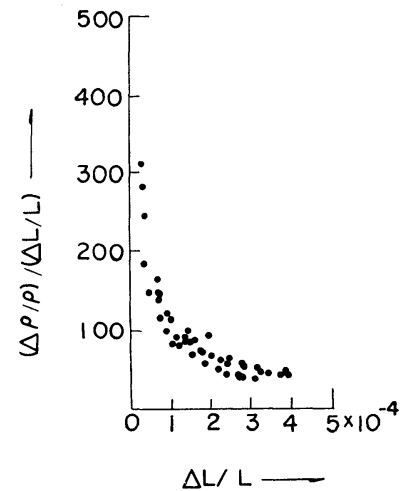

Fig. 1. $(\Delta \rho / \rho) /(\Delta L / L)$ versus $\Delta L / L$ relation for a lapilli tuff specimen from Aburatsubo. smaller than $10^{-4}$. Since the water permeability of the rock specimen is very large, it is suspected that very many small pores filled with

*) Earthquake Research Institute, University of Tokyo. 
conducting water are present in the specimen and that the contact area between neighbouring pores increase when the specimen is compressed.

The $(\Delta \rho / \rho) /(\Delta L / L)$ value for other rocks are mostly one order of magnitude lower than that for lapilli tuff from Aburatsubo. It has even been found that, contrary to the lapilli tuff specimen, a number of rock samples have negative $\Delta \rho / \Delta L$ values. It is also interesting to note that for a black schist specimen from Kawayama, Yamaguchi Prefecture, $\Delta \rho / \Delta L<0$ while the compression is not large and that $|\Delta \rho / \Delta L|$ decreases with further compression finally turning into the state of $\Delta \rho / \Delta L>0$, the whole range of compression being only a few bars.

Brace and others (Brace et al, 1965; Brace and Orange, 1966) measured changes in electric resistivity of rocks saturated with water under high hydrostatic pressures up to $10 \mathrm{~kb}$. A typical feature for granite is an increase in resistivity by a factor of $10^{2}$ or more with increasing pressure from zero to $10 \mathrm{~kb}$. Such a tremendous increase in resistivity is understood by supposing a forced shutting or closure of crack pores in the rock which form the conducting paths of electricity. Further increases in linear stress result in a considerable decrease in resistivity which lasts until the specimen finally breaks. The decrease that begins to be observed at a stress amounting to about one-third of the breaking one is interpreted by assuming developments of new cracks before rupture.

Granting that the above mechanism holds good for most rocks, the characteristic behaviour of the Aburatsubo tuff and similar rocks may be understood on assuming that the rocks are already in the final state, in which resistivity decreases with increasing stress, even at zero pressure.

A resistivity variometer for field use. The large rate of resistivity change as revealed by the laboratory experiments on the lapilli tuff specimen from Aburatsubo led the writers to set up a resistivity variometer at the Aburatsubo Geophysical Observatory where observations of crustal deformation by tiltmeters and extensometers have been continued over a period of 20 years. It has been found there that a crustal strain of the order of $10^{-6}$ is produced by tidal loading. It is therefore anticipated that a rate of resistivity change amounting to $10^{-3}$ or thereabouts would be observed there provided the $\Delta \rho / \rho-\Delta L / L$ relation as obtained in the laboratory experiments holds good also in the field.

The resistivity variometer designed and constructed by the writers is based on the method essentially similar to what is called 


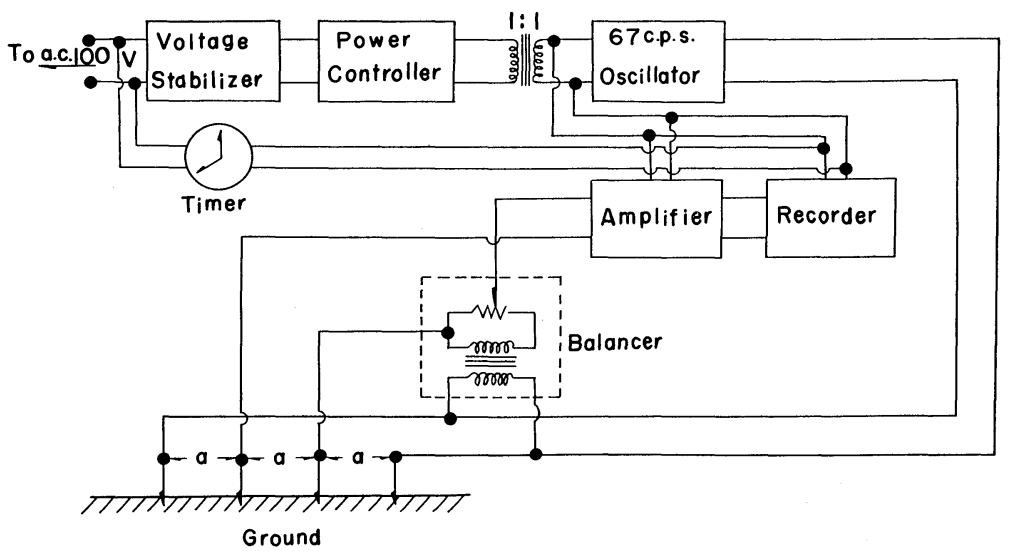

Fig. 2. Block diagram of the resistivity variometer.

the four-pole method in electric prospecting. Fig. 2 is a block diagram of the measuring circuits. A highly stable A.C. voltage of $450 \mathrm{~V}$ and 67 c.p.s. is provided by an oscillator consisting of a highly stable quartz oscillator and a power amplifier. An electric current usually amounting to some $100 \mathrm{~mA}$ is then driven into the ground through outer two of the equally spaced copper electrodes. In that case, it is well known that the relation

$$
\rho=2 \pi a V / I
$$

holds good, where $a$ is the distance between the electrodes measured in $\mathrm{cm}$, the resistivity in ohm-cm, $V$ the voltage in volt and $I$ the electric current in ampere.

The voltage picked up by the inner two electrodes is almost perfectly balanced by that taken from the oscillator through a coupling transformer. It has been proved by a circuit analysis (Yamazaki, 1965) that a small deviation voltage from the equilibrium state is proportional to the change in resistivity. Such a deviating voltage is led to an amplifier which contains a mechanical filter at 67 c.p.s.

It is important to make use of an odd frequency like 67 c.p.s. for avoiding possible contamination by the commercial A.C. of 50 c.p.s. and other man-made disturbances. The $Q$-factor of the mechanical filter is so high that these undesirable noise voltages can be eliminated to a great extent. The final output from the amplifier is easily recorded by a pen-writing recorder.

Results of the field test. The electrodes of the resistivity variometer are set in a cave in the $\mathrm{N} 81^{\circ} \mathrm{W}$ direction which is parallel to one of the working linear extensometers. The distance between the successive electrodes is $1.6 \mathrm{~m}$. The measuring site is about $10 \mathrm{~m}$ distant from the sea-shore and $3 \mathrm{~m}$ high from the mean sea level. 

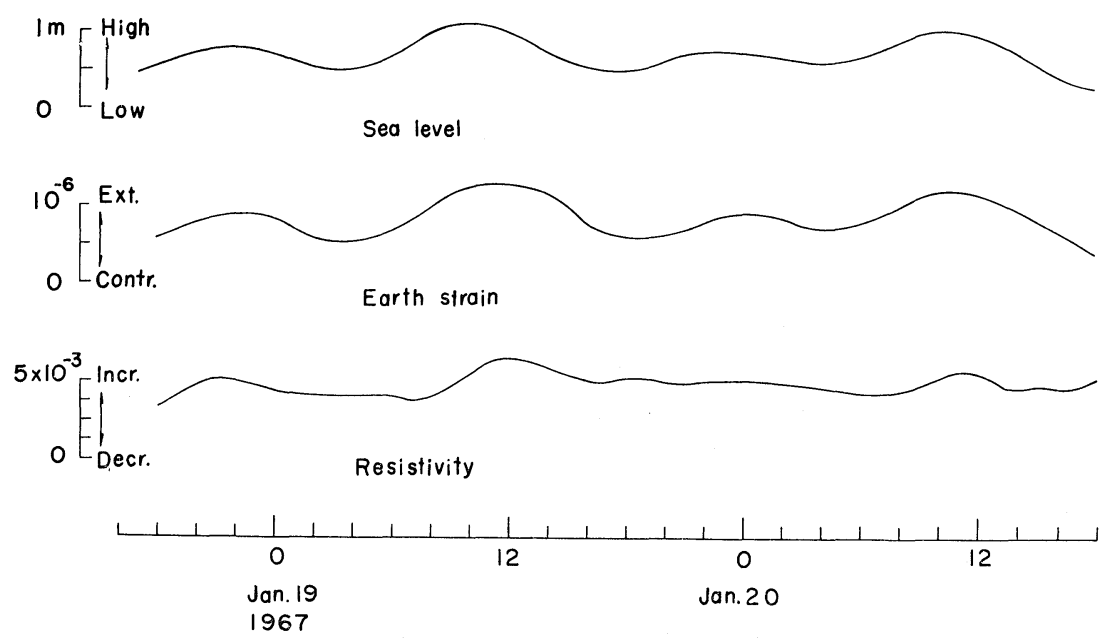

Fig. 3. Simultaneous records of changes in the sea level, earth strain and resistivity at Aburatsubo.

Fig. 3 shows an example of simultaneous records of the changes in the sea level, earth strain and rate of resistivity change as observed from Jan. 18 to 20,1967 . We can see in the figure that the earth resistivity changes almost parallel to the sea level height as well as to the earth strain. The most important point is the fact that the rate of resistivity change amounts to the order of $10^{-3}$ in contrast to the strain which amounts only to the order of $10^{-6}$. The mean value of $(\Delta \rho / \rho) /(\Delta L / L)$ is estimated as $2 \times 10^{3}$ which is larger by one order of magnitude than the one obtained from the laboratory experiment on the rock specimen taken from exactly the same place. As is seen in Fig. 1, $(\Delta \rho / \rho) /(\Delta L / L)$ increases as $\Delta L / L$ gets smaller, so that the value obtained from the field observation is not unreasonable because the observation is made under a strain which is one order of magnitude smaller than that of the laboratory experiment.

Questions may naturally be raised about the influence of the high-conducting sea-water on the resistivity change observed. At the time of high tide, the underground water-level in the permeable rocks would be raised to some extent, so that it would more or less affect the apparent crustal resistivity. In that case, however, a decrease should be observed in resistivity. This is not the case as can be seen in Fig. 3. An additional observation with electrodes buried in the ceiling of the cave also records similar changes in resistivity. Since most of electric currents are in this case flowing in rocks far from the underground water, the writers believe that the change in resistivity is actually caused by the deformation of 
the rocks around the electrodes.

Seiche oscillations of the sea water of about 13 minutes in period are sometimes observed in Aburatsubo Bay. Changes in resistivity having the same period are also observed by rapid-run recordings. The amplitude of the seiche being about one-tenth of that of the tide, say $10 \mathrm{~cm}$ or so, it is seen that the seiche causes an earth strain of the order of $10^{-7}$. It is interesting to note that the resistivity variometer can record changes associated with such a small strain although the detection is possible only after applying a low-pass filter to the original record in order to eliminate superposed noises.

Discussion and future problems. The fact that the rate of resistivity change is about $10^{3}$ times as large as the earth strain has an extremely important bearing in connection with detection of very small deformation of the earth crust. Should the sensitivity of measuring the resistivity change can be raised to $10^{-6}$, it would not be impossible to detect a strain of the order of $10^{-9}$ or thereabouts. It is most important to point out that, in so far as changes in electrical conductivity due to mechanical strain is concerned, a role of amplifier is played by the medium of the earth crust per se.

The present resistivity variometer is not entirely free from noises of unknown origin. Part of the noises is supposed to be caused by an interference between the electric currents used for the measurement and the commercial A.C. which is earthed somewhere. It is necessary to make the signal-to-noise ratio drastically larger in order to conduct observations of high sensitivity. The writers will attempt to refine the instrument so as to make observations of astronomical earth-tides and premonitory strain before earthquakes possible. It is also important to make the instrument highly stable in order to carry out routine observations over a long period of time.

In conclusion the writers wish to express their hearty thanks to Professor C. Tsuboi for his encouragement in this study.

\section{References}

Brace, W. F., and Orange, A. S.: Electrical resistivity changes in saturated rock under stress. Science, 153, 1529-1531 (1966).

Brace, W. F., Orange, A. S., and Madden, T. R.: The effect of pressure on the electrical resistivity of water-saturated crystalline rocks. J. Geophys. Res., 70, 5669-5678 (1965).

Rikitake, T.: A five-year plan for earthquake prediction research in Japan. J. Tectonophys., 3, 1-15 (1966). 
Yamazaki, Y.: Electrical conductivity of strained rocks. The first paper. Laboratory experiments on sedimentary rocks. Bull. Earthquake Res. Inst., 43, 783-802 (1965).

-: Electrical conductivity of strained rocks. The second paper. Further experiments on sedimentary rocks. Bull. Earthquake Res. Inst., 44, 1553-1570 (1966). 